\title{
SYSTEMS OF TOEPLITZ OPERATORS ON $H^{2}$. II
}

\author{
BY \\ H. R. POUSSON(1)
}

1. Introduction. Let $L^{p}(0<p \leqq \infty)$ be the usual Lebesgue space with respect to normalized Lebesgue measure on the unit circle. The space $H^{p}(0<p \leqq \infty)$ will consist of analytic functions $f$ on the unit disc such that $\lim _{r \rightarrow 1-}\left\|f\left(r e^{i \theta}\right)\right\|_{p}<\infty$. If $f \in H^{p}$, then the function defined a.e. by $f\left(e^{i \theta}\right)=\lim _{r \rightarrow 1} f\left(r e^{i \theta}\right)$ is in $L^{p}$. Should $p \geqq 1$, such functions in $L^{p}$ are precisely those which have vanishing negative Fourier coefficients. The subspace of functions $f \in H^{p}$ such that $f(0)=0$ is denoted by $H_{0}^{p}$.

The space of $n$ by $m$ dimensional, matrix-valued functions with terms in $L^{p}$ is denoted by $L_{n \times m}^{p}$. The spaces $H_{n \times m}^{p}$ and $H_{0 ; n \times m}^{p}$ are defined analogously.

Let $\phi \in L_{n \times n}^{\infty}$. Define the operator $T_{\phi}: H_{n \times l}^{2} \rightarrow H_{n \times l}^{2}$ by setting $T_{\phi} f=P \phi f$ for all $f \in H_{n \times l}^{2}$ where $P: L_{n \times m}^{2} \rightarrow H_{n \times m}^{2}$ is the projection operator. The operator $T_{\phi}$ can be considered as a system of Toeplitz operators on $H^{2}$, and $T_{\phi}$ will be called the Toeplitz operator associated with the matrix-valued function $\phi$.

This paper concerns conditions on $\phi \in L_{n \times n}^{\infty}$ which give an invertible $T_{\phi}$. After defining suitable norms for matrix functions, we consider the problem of angle between manifolds of matrix functions. This gives results which are applicable to systems of Toeplitz operators. The approach is similar to the method used by Devinatz [2] for $\phi$ scalar.

2. Definitions and general results. Most of the preliminary results are stated without proof. The proofs are available in the literature or require only a simple generalization of proofs in the literature.

Denote normalized Lebesgue measure on the unit circle by $d \mu$.

For $F$ a matrix function, let $F_{k}=\int F\left(e^{i \theta}\right) e^{-i k \theta} d \mu ; k=0, \pm 1, \pm 2, \ldots$ Denote the conjugate transpose, transpose, and complex conjugate of $F$ by $F^{*}, F^{\prime}$, and $\bar{F}$ respectively. A matrix $J$ will be called a projection matrix provided $J=J^{2}=J^{*}$.

Definition 2.1. A function $F \in H_{n \times n}^{p}$ is outer provided $\int \log |\operatorname{det} F| d \mu=$ $\log \left|\operatorname{det} F_{0}\right|>-\infty$. A function $F \in H_{n \times n}^{\infty}$ is inner provided $F=J U$ where $J$ is a constant projection matrix and $U$ is unitary a.e.

Thus $F \in H_{n \times n}^{p}$ is outer if and only if $\operatorname{det} F \in H^{p / n}$ is outer.

THEOREM 2.2. If $F \in H_{n \times n}^{2}$ is outer, and if $F^{-1} \in L_{n \times n}^{2}$, then $F^{-1} \in H_{n \times n}^{2}$.

Received by the editors April 28, 1967.

(1) The results of this paper are contained in the author's doctoral dissertation, University of Virginia, 1967. This work was partially supported by the National Science Foundation under grant NSF GP-3445. 
A proof of the following is contained in [8].

THEOREM 2.3. If $F, F^{-1} \in H_{n \times n}^{2}$, then $F$ is an outer function.

THEOREM 2.4. If $W \in L_{n \times n}^{1}$ is hermitian positive semidefinite, then $W$ has a factorization $W=B B^{*}$ where $B \in H_{n \times n}^{2}$ is outer provided $\int \log \operatorname{det} W d \mu>-\infty$.

For a proof of the above, see [5, p. 193].

For $F \in H_{n \times n}^{p}, S(F)$ will denote the subspace of $H_{n \times n}^{p}$ spanned by $\left\{F e^{i k \theta}\right\}_{k=0}^{\infty}$. The following is proved by Masani [7, p. 286].

THEOREM 2.5. Let $K \in H_{n \times n}^{2} ; K \neq 0$. Then $K=F G$ where $F \in H_{n \times n}^{2}$ is outer and $G \in H_{n \times n}^{\infty}$ is inner. Also $S(G)=S(K)$.

It can be shown that the Hilbert space adjoint $T_{\phi}^{*}$ of $T_{\phi}$ is $T_{\phi^{*}}$; hence $T_{\phi}$ is invertible if and only if $T_{\phi^{*}}$ is invertible.

THEOREM 2.6. Let $\phi \in L_{n \times n}^{\infty}$. In order for $T_{\phi}$ to be invertible, it is necessary and sufficient that

(i) $\phi=G^{*} H$ where $G, G^{-1}, H, H^{-1} \in H_{n \times n}^{2} ; S(G)=S(H)=H_{n \times n}^{2}$; and

(ii) $K: f \rightarrow H^{-1} P\left(G^{-1}\right)^{*} f$ defines a bounded operator from $L_{n \times l}^{2}$ to $H_{n \times l}^{2}$.

The proof of the above theorem and the proofs of the remaining results in this section are contained in [8].

Corollary 2.7. If $T_{\phi}$ is invertible, then $\phi^{-1} \in L_{n \times n}^{\infty}$.

THEOREM 2.8. Suppose $\phi \in L_{n \times n}^{\infty}$ is positive definite. The following are equivalent.

(i) $T_{\phi}$ is invertible.

(ii) ess inf det $\phi>0$.

(iii) $T_{\operatorname{det} \phi}$ is invertible.

REMARK 2.9. If $F \in H_{n \times n}^{\infty}$, then $T_{\phi} T_{F}=T_{\phi F}$ and $T_{F *} T_{\phi}=T_{F^{*} \phi}$.

THEOREM 2.10. Let $\phi \in H_{n \times n}^{\infty}$, then $T_{\phi}$ is invertible if and only if $\phi^{-1} \in H_{n \times n}^{\infty}$ in which case $T_{\phi}^{-1}=T_{\phi}^{-1}$.

THEOREM 2.11. If $\phi \in L_{n \times n}^{\infty}$ and $T_{\phi}$ is invertible, then there exists a factorization $\phi=U K$ where $U$ is unitary, $K \in H_{n \times n}^{\infty}$ is outer, and both $T_{K}$ and $T_{U}$ are invertible.

REMARK 2.12. If $\phi \in L_{n \times n}^{\infty}$ is unitary, then $T_{\phi}$ invertible implies $\phi=G^{*} H$ where $G, G^{-1}, H, H^{-1} \in H_{n \times n}^{2}$ and $H H^{*}=\left(G G^{*}\right)^{-1}$.

Proof. Consider Theorem 2.6 and use the fact that $\phi^{*}=\phi^{-1}$.

3. Matrix norms. Unless otherwise stated, all matrix functions will be $n$ by $n$ dimensional.

It follows from basic matrix theory that a matrix function $F$ has a polar factorization $F=U H$ where $U$ is unitary and $H$ is positive semidefinite. Should det $F \neq 0$ a.e., the factorization is unique and $H$ is positive definite. It will be desirable to consider 
polar factorizations where the hermitian factor is on the left of the unitary factor. When necessary to distinguish the two factorizations, subscripts will be used; i.e., $F=U_{R} H_{R}=H_{L} U_{L}$.

Two matrix functions $F$ and $G$ are unitarily equivalent, denoted $F \sim G$, provided there exists a unitary matrix function $V$ such that $V^{*} G V=F$.

LEMMA 3.1. If $U$ is a unitary matrix function and if $H$ is a positive definite hermitian matrix function, then $|\operatorname{tr} U H| \leqq \operatorname{tr} H$.

Proof. There exists a unitary function $V$ such that $V^{*} U V=E$ is diagonal and $\left|e_{i, i}\right|=1$ a.e. for $i=1,2, \ldots, n$. Let $V^{*} H V=K$. Then $|\operatorname{tr} U H|=|\operatorname{tr} E K|$ $=\left|\sum_{i=1}^{n} e_{i, i} k_{i, i}\right| \leqq \operatorname{tr} K=\operatorname{tr} H$.

LEMMA 3.2. If $F=U_{R} H_{R}=H_{L} U_{L}$ are the polar factorizations of $F$, then $\operatorname{tr} H_{R}$ $=\operatorname{tr} H_{L}$.

Proof. Applying 3.1 we have $\operatorname{tr} H_{L}=\operatorname{tr} U_{L}^{*} U_{R} H_{R} \leqq \operatorname{tr} H_{R}$ and $\operatorname{tr} H_{R}=\operatorname{tr} U_{L} U_{R}^{*} H_{L}$ $\leqq \operatorname{tr} H_{L}$.

Definition 3.3. For all $F \in L_{n \times n}^{1}$, define the norm of $F$, denoted $\|F\|_{1}$, as $\|F\|_{1}=\int \operatorname{tr}\left(F F^{*}\right)^{1 / 2} d \mu$.

The properties of this norm, including the necessary triangle inequality, will be given by the next two lemmas.

LEMMA 3.4. Let $F \in L_{n \times n}^{1}$ and let $F=U_{R} H_{R}=H_{L} U_{L}$ be polar factorizations of $F$. Then $\|F\|_{1}=\int \operatorname{tr} H_{R} d \mu=\int \operatorname{tr} H_{L} d \mu$.

Proof. We have

$$
\|F\|_{1}=\int \operatorname{tr}\left(F F^{*}\right)^{1 / 2} d \mu=\int \operatorname{tr}\left(U_{R} H_{R}^{2} U_{R}^{*}\right)^{1 / 2} d \mu=\int \operatorname{tr} U_{R} H_{R} U_{R}^{*} d \mu=\int \operatorname{tr} H_{R} d \mu .
$$

The conclusion now follows from 3.2.

If $F \in L_{n \times n}^{1}$ and $U$ is unitary, then as an immediate consequence of 3.4, $\|U F\|_{1}$ $=\|F\|_{1}=\|F U\|_{1}$ and $\left\|F^{*}\right\|_{1}=\|F\|_{1}$.

LEMMA 3.5. If $F, G \in L_{n \times n}^{1}$, then $\|F+G\|_{1} \leqq\|F\|_{1}+\|G\|_{1}$.

Proof. Consider polar factorizations $U_{F+G} H_{F+G}, U_{F} H_{F}$ and $U_{G} H_{G}$ of $F+G, F$ and $G$ respectively. Since $U_{F+G} H_{F+G}=U_{F} H_{F}+U_{G} H_{G}$, it follows from 3.4 and 3.1 that

$$
\begin{aligned}
\|F+G\|_{1} & =\int \operatorname{tr} H_{F+G} d \mu \leqq \int\left|\operatorname{tr} U_{F+G}^{*} U_{F} H_{F}\right| d \mu+\int\left|\operatorname{tr} U_{F+G}^{*} U_{G} H_{G}\right| d \mu \\
& \leqq \int \operatorname{tr} H_{F} d \mu+\int \operatorname{tr} H_{G} d \mu=\|F\|_{1}+\|G\|_{1} .
\end{aligned}
$$

The Euclidean norm, denoted $|A|$, of a constant matrix $A$ is defined as $|A|$ 
$=\left(\operatorname{tr} A A^{*}\right)^{1 / 2}$. For proof that this is actually a norm, see $[9$, p. 125]. It is easily established that for constant matrices $A$ and $B,|A B| \leqq|A||B|$.

Definition 3.6. For all $F \in L_{n \times n}^{2}$, define the norm of $F$, denoted $\|F\|_{2}$, as $\|F\|_{2}=\left(\int \operatorname{tr} F F^{*} d \mu\right)^{1 / 2}=\left(\int|F|^{2} d \mu\right)^{1 / 2}$.

LEMMA 3.7. If $F, G \in L_{n \times n}^{2}$, then $\left|\int \operatorname{tr} F G d \mu\right| \leqq\|F\|_{2}\|G\|_{2}$.

Proof. It follows from Schwarz's inequality that

$$
\begin{aligned}
\left|\int \operatorname{tr} F G d \mu\right| & =\left|\sum \int f_{i, j} g_{j, i} d \mu\right| \leqq \int\left(\sum\left|f_{i, j}\right|\left|g_{j, i}\right|\right) d \mu \\
& \leqq \int\left(\sum\left|f_{i, j}\right|^{2}\right)^{1 / 2}\left(\sum\left|g_{j, i}\right|^{2}\right)^{1 / 2} d \mu \\
& \leqq\left(\int \operatorname{tr} F F^{*} d \mu\right)^{1 / 2}\left(\int \operatorname{tr} G G^{*} d \mu\right)^{1 / 2}=\|F\|_{2}\|G\|_{2} ; \quad 1 \leqq i, j \leqq n .
\end{aligned}
$$

LEMMA 3.8. If $F, G \in L_{n \times n}^{2}$, then $\|F G\|_{1} \leqq\|F\|_{2}\|G\|_{2}$.

Proof. Let $F \in L_{n \times n}^{2}$. Assume, without loss of generality, that $F^{-1} \in L_{n \times n}^{2}$. Applying 3.7, we have

$$
\|F G\|_{1}=\int \operatorname{tr}\left(F F^{*}\right)^{1 / 2}\left(F G G^{*} F^{*}\right)^{1 / 2}\left(F F^{*}\right)^{-1 / 2} d \mu \leqq\|F\|_{2}\|G\|_{2} .
$$

THEOREM 3.9. Products of the form $M N$ where $M$ is in the unit ball of $H_{n \times n}^{2}$ and $N$ is in the unit ball of $H_{0 ; n \times n}^{2}$ are dense in the open unit ball of $H_{0 ; n \times n}^{1}$.

Proof. Products of the form stated are contained in the unit ball of $H_{0 ; n \times n}^{1}$, see 3.8.

Now consider $F \in H_{0 ; n \times n}^{1}$ such that $\|F\|_{1}<1$ and $\int \log |\operatorname{det} F| d \mu>-\infty$. From 2.4, $\left(F F^{*}\right)^{1 / 2}=B B^{*}$ where $B \in H_{n \times n}^{2}$ is outer. Also, $\left\|B^{-1} F\right\|_{2}^{2}=\|B\|_{2}^{2}=\|F\|_{1}<1$. Hence $B$ is contained in the unit ball of $H_{n \times n}^{2}$ and $B^{-1} F$ is contained in the unit ball of $L_{n \times n}^{2}$. By definition, $B \in H_{n \times n}^{2}$ is outer if and only if $\operatorname{det} B$ is outer. Hence det $B$, considered as an analytic function in the open disc, does not vanish inside the disc. From this it follows that $B^{-1}$ can be considered as an analytic function in the open disc. Clearly $B^{-1} F$ has an analytic extension inside the disc which vanishes at zero, since $F_{0}=0$. Since $B^{-1} F \in L_{n \times n}^{2}$ on the unit circle, $B^{-1} F \in H_{0 ; n \times n}^{2}$. Let $M=B$ and $N=B^{-1} F$.

Consider $F \in H_{0 ; n \times n}^{1}$ such that $\|F\|_{1}<1$ and $\int \log |\operatorname{det} F| d \mu=-\infty$. Let $K$ $=e^{-t \theta} F$, then $|\operatorname{det} K|=|\operatorname{det} F|$ and $F_{1}=K_{0}$. Using a form of Jensen's inequality, see [11, p. 123], $\log \left|\operatorname{det} K_{0}\right| \leqq \int \log |\operatorname{det} K| d \mu=-\infty$. Therefore $F_{1}$ is singular.

Choose $\alpha>0$ such that $F_{1}+\alpha$ is nonsingular and $\left\|F_{\alpha}\right\|_{1}<1$ where $F_{\alpha}=F+\alpha e^{i \theta}$. Jensen's inequality implies $\int \log \left|\operatorname{det} F_{\alpha}\right| d \mu>-\infty$. It follows that functions $F$ in the unit ball of $H_{0 ; n \times n}^{1}$ for which $\int \log |\operatorname{det} F| d \mu>-\infty$ are dense in the open unit ball of $H_{0 ; n \times n \text {. }}^{1}$ 
The Banach norm, denoted $|A|_{B}$, of a constant matrix $A$ is defined as $|A|_{B}$ $=\sup _{|X| \neq 0}|A X| /|X|$.

For an $n$ by $n$ matrix $A$, the Banach norm and the Euclidean norm are related by $|A|_{B} \leqq|A| \leqq n|A|_{B}$.

LEMMA 3.10. If $A$ is a constant matrix and if $A=U H$ is its polar factorization then $|A|_{B}=d_{1,1}$ where $D$ is diagonal, $d_{1,1} \geqq d_{2,2} \geqq \cdots \geqq d_{n, n}$ and $H \sim D$.

Proof. Let $X$ be any nonzero constant matrix and let $V$ denote the unitary matrix such that $V^{*} H V=D$. Then $|A X|=\left(\operatorname{tr} H^{2} X X^{*}\right)^{1 / 2}=\left(\operatorname{tr} D^{2} V^{*} X X^{*} V\right)^{1 / 2}$ $\leqq d_{1,1}\left(\operatorname{tr} X X^{*}\right)^{1 / 2}=d_{1,1}|X|$ and $|A|_{B} \leqq d_{1,1}$.

Let $E$ be the projection matrix which has every term zero with the exception of $e_{1,1}=1$. Then

$|A V E|=\left(\operatorname{tr} U H V E V^{*} H U^{*}\right)^{1 / 2}=\left(\operatorname{tr} V^{*} H^{2} V E\right)^{1 / 2}=\left(\operatorname{tr} D^{2} E\right)^{1 / 2}=d_{1,1}=d_{1,1}|V E|$ and $|A|_{B} \geqq d_{1,1}$.

Definition 3.11. For all $F \in L_{n \times n}^{\infty}$, define the norm of $F$, denoted $\|F\|_{\infty}$, as $\|F\|_{\infty}=\operatorname{ess} \sup _{\theta}\left|F\left(e^{i \theta}\right)\right|_{B}$.

As an immediate consequence of 3.10 we have the following.

Note 3.12. Let $F \in L_{n \times n}^{\infty}$, let $F=U H$ be its polar factorization and let $H \sim D$ where $D$ is diagonal with $d_{1,1} \geqq d_{2,2} \geqq \cdots \geqq d_{n, n}$. Then $\|F\|_{\infty}=\left\|d_{1,1}\right\|_{\infty}$.

THEOREM 3.13. Suppose $G \in L_{n \times n}^{\infty}$. The linear functional $I(\cdot)$ on $L_{n \times n}^{1}$ defined for all $F \in L_{n \times n}^{1}$ by $I(F)=\int \operatorname{tr} F G d \mu$ is bounded and $\|I\|=\|G\|_{\infty}$. All bounded linear functionals on $L_{n \times n}^{1}$ have this form.

Proof. Let $U_{G} H_{G}$ be the polar factorization of $G \in L_{n \times n}^{\infty}$ and let $V$ denote the unitary matrix function such that $V^{*} H_{G} V=D$ where $D$ is diagonal, $d_{1,1} \geqq d_{2,2}$ $\geqq \cdots \geqq d_{n, n}$. Suppose $U_{F} H_{F}$ is the polar factorization of an arbitrary $F \in L_{n \times n}^{1}$. Set $K=V^{*} U_{F} H_{F} U_{G} V$ and let $J$ be the following diagonal (unitary) matrix. If $k_{i, i} \neq 0$, set $j_{i, i}=k_{i, i}\left|k_{i, i}^{-1}\right|$, and if $k_{i, i}=0$, set $j_{i, i}=1 ; i=1,2, \ldots, n$. Then

$$
\begin{aligned}
|I(F)| & =\left|\int \operatorname{tr} U_{F} H_{F} U_{G} H_{G} d \mu\right|=\left|\int \operatorname{tr} K D d \mu\right| \leqq \int\left(\sum_{i=1}^{n}\left|k_{i, i}\right|\left|d_{i, i}\right|\right) d \mu \\
& \leqq\left\|d_{1,1}\right\|_{\infty} \int\left(\sum_{i=1}^{n}\left|k_{i, i}\right|\right) d \mu=\|G\|_{\infty} \int \operatorname{tr} J K d \mu \\
& =\|G\|_{\infty} \int \operatorname{tr} U_{G} V J V^{*} U_{F} H_{F} d \mu \leqq\|G\|_{\infty} \int \operatorname{tr} H_{F} d \mu=\|G\|_{\infty}\|F\|_{1} .
\end{aligned}
$$

Therefore $I(\cdot)$ is bounded and $\|I\| \leqq\|G\|_{\infty}$.

Now for $\alpha>0$, take $E=\left[\left.e^{i \theta}|| G\left(e^{i \theta}\right)\right|_{B} \geqq\|G\|-\alpha\right]$. Then $\mu(E)=\int \chi_{E} d \mu \neq 0$ where $\chi_{E}$ is the characteristic function of $E$.

Let $Q$ denote the projection matrix which has every term equal to zero with the 
exception of $q_{1,1}=1$. Set $F_{\alpha}=Q \chi_{E} / u(E)$, then $\left\|F_{\alpha}\right\|_{1}=1$. If $K_{\alpha}=V^{*} F_{\alpha} V U_{G}^{*}$, then $\left\|K_{\alpha}\right\|_{1}=\left\|F_{\alpha}\right\|_{1}=1$ and

$$
\begin{aligned}
I\left(K_{\alpha}\right) & =\int_{E} \operatorname{tr}\left[\frac{V^{*} Q V U_{G}^{*}}{u(E)} \cdot G\right] d \mu=\frac{1}{u(E)} \int_{E} \operatorname{tr} V^{*} Q V H_{G} d \mu \\
& =\frac{1}{u(E)} \int_{E} \operatorname{tr} J D d \mu=\frac{1}{u(E)} \int_{E}|G|_{B} d \mu .
\end{aligned}
$$

Hence $\|G\|_{\infty}-\alpha \leqq\left|I\left(K_{\alpha}\right)\right| \leqq\|I\|$. Thus $\|G\|_{\infty}=\|I\|$.

It can be deduced from the analogous result for the scalar case that all bounded linear functionals on $L_{n \times n}^{1}$ have the form asserted.

4. Manifolds of matrix functions. Let $W \in L_{n \times n}^{1}$ be positive definite hermitian. Denote by $L_{n \times n}^{2}(W)$ the space of matrix functions $F$ for which $\|F\|_{W}^{2}=\int \operatorname{tr} F F^{*} W d \mu$ $<\infty$. This is a Hilbert space with inner product defined by $(F, G)_{W}=\int \operatorname{tr} F G^{*} W d \mu$ for all $F, G \in L_{n \times n}^{2}(W)$.

Consider $F \in L_{n \times n}^{2}(W)$ such that $F$ is orthogonal to the trigonometric polynomials, all of which are clearly contained in $L_{n \times n}^{2}(W)$. Then for all constant matrices $C$, and $k=0, \pm 1, \pm 2, \ldots,\left(e^{i k \theta} C, F\right)_{\mathrm{W}}=\int \operatorname{tr} e^{i k \theta} C F^{*} W d \mu=0$. Since $\left\|F^{*} W^{1 / 2}\right\|_{2}^{2}=\|F\|_{W}^{2}, F^{*} W^{1 / 2} \in L_{n \times n}^{2}$, so $F^{*} W \in L_{n \times n}^{1}$ and has a Fourier series expansion. Thus $F^{*} W=0$. If $\operatorname{det} W \neq 0$ a.e., then $F^{*}=0$ a.e. Hence the trigonometric polynomials are dense in $L_{n \times n}^{2}(W)$ provided det $W \neq 0$ a.e.

Denote by $\Delta_{n \times n}$ the space of all analytic trigonometric polynomialš with $n$ by $n$ constant matrix coefficients. Let $\Delta_{0 ; n \times n}$ be the subspace of all $A \in \Delta_{n \times n}$ for which $A_{0}=0$. Denote by $H_{n \times n}^{2}(W)$ and $H_{0 ; n \times n}^{2 \times}(W)$ the subspaces of $L_{n \times n}^{2}(W)$ generated by $\Delta_{n \times n}$ and $\Delta_{0 ; n \times n}$ respectively. Let $\bar{H}_{0 ; n \times n}^{2}(W)$ be the subspace of $L_{n \times n}^{2}(W)$ generated by all conjugate transposes of elements of $\Delta_{0 ; n \times n}$.

DeFINITION 4.1. Let

$$
\delta=\sup \left[\left|\left(F, K^{*}\right)_{w}\right| \mid F \in H_{n \times n}^{2}(W) ; K^{*} \in \bar{H}_{0 ; n \times n}^{2}(W) ;\|F\|_{w} \leqq 1 ;\left\|K^{*}\right\|_{w} \leqq 1\right] \text {. }
$$

The manifolds $H_{n \times n}^{2}(W)$ and $\bar{H}_{0 ; n \times n}^{2}(W)$ are at positive angle provided $\delta<1$.

Consider a scalar function $w \in L^{1}$. If $\log w$ is summable, then there exists an outer function $h \in H^{1}$ such that $|h|=w$. Helson and Szego [6] have proved the following.

THEOREM 4.2. The manifolds $H^{2}(w)$ and $\bar{H}_{0}^{2}(w)$ are at positive angle if and only if there exists $g \in H^{\infty}$ and $\alpha>0$ such that $\left|g\left(e^{i \theta}\right)\right| \geqq \alpha$ a.e. and $|\arg h g| \leqq \pi / 2-\alpha$.

A matrix form of this result will be shown to hold.

If $A$ and $B$ are hermitian, then $A \leqq B$ provided $B-A$ is positive semidefinite.

Let $W \in L_{n \times n}^{1}$ be positive definite hermitian and suppose $\log$ det $W$ is summable. Then there exists two factorizations of $W$; that is, $W=A^{*} A=B B^{*}$ where $A, B$ $\in H_{n \times n}^{2} ; A$ and $B$ are both outer functions. Let $U=B^{-1} A^{*}=B^{*}\left(A^{-1}\right)$. Then $U$ is unitary and $W=B U A$. In the theorem and proof which follow $W, U, A$ and $B$ will be as just described. 
THEOREM 4.3. The manifolds $H_{n \times n}^{2}(W)$ and $\bar{H}_{0 ; n \times n}^{2}(W)$ are at positive angle if and only if there exists $G \in H_{n \times n}^{\infty}$ and $\alpha>0$ such that

$$
\alpha+G^{*} G \leqq U^{*} G+G^{*} U .
$$

Proof. Since $W=B U A$, we have from 4.1 that $\delta=\sup _{F, K}\left|\int \operatorname{tr} F K W d \mu\right|=$ $\sup _{F, K}\left|\int \operatorname{tr} A F K B U d \mu\right|<1$ where $F$ varies throughout the unit ball of $H_{n \times n}^{2}(W)$ and $K^{*}$ varies throughout the unit ball of $\bar{H}_{0 ; n \times n}^{2}(W)$.

For all $F \in H_{n \times n}^{2}(W)$ there exists a sequence $\left\{P_{s}\right\}_{1}^{\infty}$ of elements of $\Delta_{n \times n}$ such that $\lim _{s \rightarrow \infty}\left\|P_{s}-F\right\|_{W}=0$. For $s=1,2,3, \ldots\left\|P_{s}-F\right\|_{W}=\left\|A P_{s}-A F\right\|_{2}$, and so $\lim _{s \rightarrow \infty}\left\|A P_{s}-A F\right\|_{2}=0$. Clearly $A P_{s} \in H_{n \times n}^{2}$ for each $s$; hence $A F \in H_{n \times n}^{2}$. Moreover $\|F\|_{W}=\|A F\|_{2}$. Thus $F$ in the unit ball of $H_{n \times n}^{2}(W)$ implies $A F$ is in the unit ball of $H_{n \times n}^{2}$. In fact, since $A$ is outer, the set of all products $A F$ where $F \in H_{n \times n}^{2}(W)$, $\|F\|_{W} \leqq 1$, is dense in the unit ball of $H_{n \times n}^{2}$. A similar argument shows that the set of all products $K B$ where $K^{*} \in \bar{H}_{0 ; n \times n}^{2}(W),\left\|K^{*}\right\|_{w} \leqq 1$, is dense in the unit ball of $H_{0 ; n \times n}^{2}$. Thus it follows from 3.9 that $\delta=\sup _{R}\left|\int \operatorname{tr} R U d \mu\right|$ where $R$ varies throughout the unit ball of $H_{0 ; n \times n}^{1}$. Theorem 3.13 implies that $\int \operatorname{tr} R U d \mu$ defines a bounded linear functional on $L_{n \times n}^{1}$ which, when restricted to $H_{0 ; n \times n}^{1}$, has norm $\delta$.

Suppose $S \in L_{n \times n}^{\infty}$ is an annihilator of $H_{0 ; n \times n}^{1}$; that is, for all $R \in H_{0 ; n \times n}^{1}$, $\int \operatorname{tr} R S d \mu=0$. Necessarily, $S \in H_{n \times n}^{\infty}$. Moreover, all elements of $H_{n \times n}^{\infty}$ are annihilators of $H_{0 ; n \times n}^{1}$.

We now apply a corollary of the Hahn-Banach theorem, see [1], and obtain $\delta=\inf _{S \in H_{n \times n}^{\infty}}\|U-S\|_{\infty}$.

Choose $G \in H_{n \times n}^{\infty}$ such that $\|U-G\|_{\infty} \leqq 1-\alpha$ where $\alpha>0$. Since multiplication by a unitary matrix leaves the norm constant, $\|U-G\|_{\infty}=\left\|I-U^{*} G\right\|_{\infty} \leqq 1-\alpha$. It follows from the definition of the $L_{n \times n}^{\infty}$ norm that $\left|I-U^{*}\left(e^{i \theta}\right) G\left(e^{i \theta}\right)\right|_{B} \leqq 1-\alpha$ a.e. This implies that

$$
\left(\left[I-U^{*}\left(e^{i \theta}\right) G\left(e^{i \theta}\right)\right] X,\left[I-U^{*}\left(e^{i \theta}\right) G\left(e^{i \theta}\right)\right] X\right)^{1 / 2} \leqq 1-\alpha
$$

a.e. for any constant matrix $X$ with $|X|=1$. From this it follows that $\alpha+G^{*} G$ $\leqq U^{*} G+G^{*} U$. We could just as readily obtain that $\alpha+G G^{*} \leqq G U^{*}+U G^{*}$.

All the above steps are reversible so the theorem follows.

In order for (4.4) to hold, it is necessary that ess inf det $G>0$. In the scalar case, 4.2 and 4.3 are equivalent.

We continue to require that $\log \operatorname{det} W$ be summable.

THEOEREM 4.5. The manifolds $H_{n \times n}^{2}(W)$ and $\bar{H}_{0 ; n \times n}^{2}(W)$ are at positive angle if and only if there exists $M>0$ such that $\|F\|_{w} \leqq M\left\|F+G^{*}\right\|_{w}$ for all $F \in H_{n \times n}^{2}(W)$ and $G^{*} \in \bar{H}_{0 ; n \times n}^{2}(W)$.

A proof of the above will not be given here since it is not significantly different from that of the scalar case.

Consider a trigonometric polynomial $\sum A_{k} e^{i k \theta}$. If $H_{n \times n}^{2}(W)$ and $\bar{H}_{0 ; n \times n}^{2}(W)$ are at 
positive angle, then 4.5 implies the existence of $M>0$ such that $\left\|\sum_{k=-s}^{s} A_{k} e^{i k \theta}\right\|_{w}$ $\leqq M\left\|\sum A_{k} e^{i k \theta}\right\|_{w}$ for $s=0,1,2, \ldots$

LEMMA 4.6. If $H_{n \times n}^{2}(W)$ and $\bar{H}_{0 ; n \times n}^{2}(W)$ are at positive angle, then $F \in L_{n \times n}^{2}(W)$ implies $F \in L_{n \times n}^{1}$.

Proof. Suppose $C$ is a constant matrix such that $W_{0} C=0$. Since $\left(W_{0} C, C\right)$ $=(W C, C)=\left\|W^{1 / 2} C\right\|_{2}^{2}=0, W^{1 / 2} C=0$ which implies $C=0$. Therefore $W_{0}$ is nonsingular.

The operator which sends each trigonometric polynomial into its constant term is bounded. Hence the linear functional $I(\cdot)$ defined for all trigonometric polynomials $A$ by $I(A)=\left(A_{0}, I\right)_{W}=\operatorname{tr} A_{0} W_{0}$ is bounded on a dense subset of $L_{n \times n}^{2}(W)$. Hence there exists $K \in L_{n \times n}^{2}(W)$ such that $I(F)=(F, K)_{W}$ for all $F \in L_{n \times n}^{2}(W)$. It follows that $W K=W_{0}$, so $K W_{0}^{-1}=W^{-1} \in L_{n \times n}^{2}(W)$ and $\left\|W^{-1}\right\|_{W}^{2}=\int \operatorname{tr} W^{-1} d \mu$ $<\infty$. Applying 3.7, we obtain

$$
\|F\|_{1}=\int \operatorname{tr}\left(F F^{*}\right)^{1 / 2} W^{1 / 2} W^{-1 / 2} d \mu \leqq\left(\int \operatorname{tr} F F^{*} W d \mu\right)^{1 / 2}\left(\int \operatorname{tr} W^{-1} d \mu\right)^{1 / 2}<\infty .
$$

THEOREM 4.7. If $H_{n \times n}^{2}(W)$ and $\bar{H}_{0 ; n \times n}^{2}(W)$ are at positive angle, then the Fourier series of $F \in L_{n \times n}^{2}(W)$ converges to $F$ in norm.

Proof. For all trigonometric polynomials $\sum A_{k} e^{i k \theta}$, let $Q_{s}\left(\sum A_{k} e^{i k \theta}\right)$ $=\sum_{k=-s}^{s} A_{k} e^{i k \theta} ; s=0,1,2, \ldots$ Then $Q_{s}$ has a bounded extension to all of $L_{n \times n}^{2}(W)$. Also for any trigonometric polynomial $A,\left\|\left(Q_{s}-I\right) A\right\|_{w} \rightarrow 0$ as $s \rightarrow \infty$.

Let $\alpha>0$ and $F \in L_{n \times n}^{2}(W)$. There exists a trigonometric polynomial $F_{\alpha}$ such that $\left\|F-F_{\alpha}\right\|_{w}<\alpha$. Now

$\left\|\left(Q_{s}-I\right) F\right\|_{w} \leqq\left\|\left(Q_{s}-I\right)\left(F-F_{\alpha}\right)\right\|_{w}+\left\|\left(Q_{s}-I\right) F_{\alpha}\right\|_{w} \leqq M\left\|F-F_{\alpha}\right\|_{w}+\left\|\left(Q_{s}-I\right) F_{\alpha}\right\|_{w}$ where $M>0$. The theorem follows.

\section{Invertibility of systems of Toeplitz operators on $H^{2}$.}

TheOREM 5.1. Let $\phi=G^{*} H$ where $H, H^{-1}, G, G^{-1} \in H_{n \times n}^{2}$ and $H H^{*}=\left(G G^{*}\right)^{-1}$. If $W=G G^{*}$, then $T_{\phi}$ is invertible if and only if there exists $B>0$ such that $\|F\|_{w}$ $\leqq B\left\|F+K^{*}\right\|_{w}$ for all $F \in H_{n \times n}^{2}(W)$ and $K^{*} \in \bar{H}_{0 ; n \times n}^{2}(W)$.

Proof. Suppose $R \in H_{n \times n}^{2}$ is orthogonal to the range of $T_{\phi}$. Then for all $F \in H_{n \times n}^{2}$, $0=\left(T_{\phi} F, R\right)=\int \operatorname{tr} \phi F R^{*} d \mu=(H F, G R)$. Since $H$ is outer, all products of the form $H F, F \in H_{n \times n}^{2}$, are dense in $H_{n \times n}^{2}$. Thus $G R=0$ and $R=0$. Hence the range of $T_{\phi}$ is dense in $H_{n \times n}^{2}$.

Let $N \in H_{n \times n}^{2}$, then $N=H^{-1} F$ where $F=H N \in H_{n \times n}^{1}$. Similarly, for $M \in H_{0 ; n \times n}^{2}$, $M^{*}=G^{*} E^{*}$ where $E=M\left(G^{-1}\right) \in H_{0 ; n \times n}^{1}$. Since $H^{-1}$ is outer (see 2.3), there exists a sequence $\left\{P_{s}\right\}_{s=1}^{\infty} \subseteq \Delta_{n \times n}$ such that $H^{-1} P_{s} \in H_{n \times n}^{2}$ for $s=1,2,3, \ldots$ and $\lim _{s \rightarrow \infty}\left\|H^{-1} P_{s}-N\right\|_{2}=0$. For $s=1,2,3, \ldots,\left\|H^{-1} P_{s}-N\right\|_{2}^{2}=\left\|P_{s}-F\right\|_{W}^{2}$ so $\lim _{s \rightarrow \infty}\left\|P_{s}-F\right\|_{W}=0$ and $F \in H_{n \times n}^{2}(W)$. Similarly, $E^{*} \in \bar{H}_{0 ; n \times n}^{2}(W)$. 
Now consider $T_{\phi}\left(H^{-1} F\right)=\phi H^{-1} F-(I-P) \phi H^{-1} F$. Since $(I-P) \phi H^{-1} F$ $=G^{*} E_{r}^{*}$ for some $E_{r} \in H_{0 ; n \times n}^{1}$, the expression can be rewritten as $T_{\phi}\left(H^{-1} F\right)$ $=G^{*} F+G^{*} E_{r}^{*}$. Note that $\left\|H^{-1} F\right\|_{2}=\|F\|_{w}$. It follows that $\|N\|_{2}^{2}=\|F\|_{w}^{2} \leqq$ $B^{2}\left\|F+E_{r}^{*}\right\|_{W}^{2}=B^{2}\left\|G^{*} F+G^{*} E_{r}^{*}\right\|_{2}^{2}=B^{2}\left\|T_{\phi}\left(H^{-1} F\right)\right\|_{2}^{2}=B^{2}\left\|T_{\phi}(N)\right\|_{2}^{2}$; that is, $\|N\|_{2}$ $\leqq B\left\|T_{\phi}(N)\right\|_{2}$.

Conversely, suppose $T_{\phi}$ is invertible. Consider $F \in H_{n \times n}^{2}(W)$. There exists a sequence $\left\{P_{s}\right\}_{s=1}^{\infty} \subseteq \Delta_{n \times n}$ such that $\lim _{s \rightarrow \infty}\left\|P_{s}-F\right\|_{W}=0$. Hence $\lim _{s \rightarrow \infty} \| H^{-1} P_{s}$ $-H^{-1} F \|_{2}^{2}=0$ and $H^{-1} F \in H_{n \times n}^{2}$. Recall that $\left\|H^{-1} F\right\|_{2}=\|F\|_{w}$. Note that $G^{*} E_{r}^{*}$ is the projection of $G^{*} F$ onto $H_{n \times n}^{2}$. Now for all $E^{*} \in \bar{H}_{0 ; n \times n}^{2}(W),\|F\|_{W}^{2}=\left\|H^{-1} F\right\|_{2}^{2}$ $\leqq B^{2}\left\|T_{\phi}\left(H^{-1} F\right)\right\|_{2}^{2}=B^{2}\left\|G^{*} F+G^{*} E_{r}^{*}\right\|_{2}^{2} \leqq B^{2}\left\|G^{*} F+G^{*} E^{*}\right\|_{2}^{2}=B^{2}\left\|H^{-1} F+H^{-1} E^{*}\right\|_{2}^{2}$ $=B^{2}\left\|F+E^{*}\right\|_{W}^{2}$; that is, $\|F\|_{W} \leqq B\left\|F+E^{*}\right\|_{W}$ for some $B>0$.

LeMma 5.2. Suppose $\phi \in L_{n \times n}^{\infty}$ is unitary and $T_{\phi}$ is invertible. Then there exists $G \in H_{n \times n}^{\infty}$ and $\alpha>0$ such that $\alpha+G^{*} G \leqq \phi^{*} G+G^{*} \phi$.

Proof. It follows from 2.12 that $\phi=K^{*} H$ where $H, H^{-1}, K, K^{-1} \in H_{n \times n}^{2}$ and $H H^{*}=\left(K K^{*}\right)^{-1}$. Let $W=K K^{*}$. Then 5.1 implies that for some $B>0,\|F\|_{w}$ $\leqq B\left\|F+K^{*}\right\|_{w}$ for all $F \in H_{n \times n}^{2}(W)$ and $K^{*} \in \bar{H}_{0 ; n \times n}^{2}(W)$. From 4.5, $H_{n \times n}^{2}(W)$ and $H_{0 ; n \times n}^{2}(W)$ are at positive angle. Since $W=K \phi H^{-1}, \phi$ has the same properties relative to $W$ as $U$ in 4.3. The lemma follows.

LEMMA 5.3. Suppose $\phi \in L_{n \times n}^{\infty}$ is unitary and $\phi=K^{*} H$ where $H, H^{-1}, K, K^{-1}$ $\in H_{n \times n}^{2}$. If there exists $G \in H_{n \times n}^{\infty}$ and $\alpha>0$ such that $\alpha+G^{*} G \leqq \phi^{*} G+G^{*} \phi$, then $T_{\phi}$ is invertible.

Proof. Since $\phi$ is unitary, $H H^{*}=\left(K K^{*}\right)^{-1}$. Let $W=K K^{*}$, then $W=K \phi H^{-1}$. From 4.3, $H_{n \times n}^{2}(W)$ and $\bar{H}_{0 ; n \times n}^{2}(W)$ are at positive angle. From 4.5 , there exists $B>0$ such that $\|F\|_{w} \leqq B\left\|F+E^{*}\right\|_{w}$ for all $F \in H_{n \times n}^{2}(W)$ and $E^{*} \in \bar{H}_{0 ; n \times n}^{2}(W)$. Now 5.1 implies $T_{\phi}$ is invertible.

TheOREM 5.4. Let $\phi \in L_{n \times n}^{\infty}$ and suppose $T_{\phi}$ is invertible. Let $\phi=U K$ be the unitary-outer factorization of $\phi$ (see 2.11). Then

(i) ess inf $|\operatorname{det} \phi|>0$;

(ii) there exists $G \in H_{n \times n}^{\infty}$ and $\alpha>0$ such that $\alpha+G^{*} G \leqq U^{*} G+G^{*} U$.

Proof. For (i), see 2.7. Since 2.11 implies $T_{U}$ is invertible, the theorem follows from 5.2.

THEOREM 5.5 Let $\phi \in L_{n \times n}^{\infty}$ and suppose $\phi^{-1} \in L_{n \times n}^{\infty}$. Let $\phi=U K$ be the unitary outer factorization of $\phi$. If $U=R^{*} H$ where $R, R^{-1}, H, H^{-1} \in H_{n \times n}^{2}$, and if there exists $G \in H_{n \times n}^{\infty}$ and $\alpha>0$ such that $\alpha+G^{*} G \leqq U^{*} G+G^{*} U$, then $T_{\phi}$ is invertible.

Proof. Since $\phi^{-1} \in L_{n \times n}^{\infty}, \phi$ has a unitary-outer factorization $\phi=U K$ with $T_{K}$ invertible. From 5.3, $T_{U}$ is invertible. Since $T_{\phi}=T_{U} T_{K}, T_{\phi}$ is invertible.

In the scalar case, Devinatz [2] has shown the following. 
THEOREM 5.6. Suppose $\phi \in L^{\infty}$. Then $T_{\phi}$ is invertible if and only if

(i) ess inf $|\phi|>0$;

(ii) there exists $g \in H^{\infty}$ such that $g^{-1} \in H^{\infty}$ and $\alpha>0$ such that $|\arg g \phi| \leqq \pi / 2-\alpha$.

\section{BIBLIOGRAPHY}

1. F. F. Bonsall, Dual extremum problems in the theory of functions, J. London Math. Soc. 31 (1956), 105-110.

2. A. Devinatz, Toeplitz operator on $H^{2}$ spaces, Trans. Amer. Math. Soc. 112 (1964), 304-3.17.

3. P. Hartman and A. Wintner, The spectra of Toeplitz matrices, Amer. J. Math. 76 (1954), 867-882.

4. H. Helson, Lectures on invariant subspaces, Academic Press, New York, 1964.

5. H. Helson and D. Lowdenslager, Prediction theory and Fourier series in several variables, Acta Math. 99 (1958), 165-202.

6. H. Helson and G. Szego, A problem in prediction theory, Ann. Mat. Pura Appl. 51 (1960), 107-138.

7. P. Masani, Shift invariant spaces and prediction theory, Acta Math. 107 (1962), 275-290.

8. H. Pousson, Systems of Toeplitz operators on $H^{2}$, Proc. Amer. Math. Soc. 19 (1968), 603-608.

9. J. H. M. Wedderburn, Lectures on matrices. Amer. Math. Soc. Colloq. Publ., vol. 17, Amer. Math. Soc., Providence, R. I., 1934.

10. H. Widom, Inversion of Toeplitz matrices, II, Illinois J. Math. 4 (1960), 88-99.

11. N. Wiener and P. Masani, Multivariate stochastic processes. I, Acta Math. 98 (1957), 111-150.

College of William and Mary,

Williamsburg, Virgina

UNIVERSTTY OF KENTUCKY,

LEXINGTON, KeNTUCKY 